\title{
Optimizing the Efficiency and Implementation of Cash Transfers to Improve Adherence to Antiretroviral Therapy: A Cluster Randomized Controlled Trial (Study Protocol)
}

\section{Laura Packel ( $\sim$ lpackel@berkeley.edu )}

University of California Berkeley School of Public Health https://orcid.org/0000-0002-8186-5246

\section{Prosper Njau}

Ministry of Health Community Development Gender Elderly and Children

\section{Carolyn Fahey}

University of California Berkeley School of Public Health

\section{Angela Ramadhani}

Ministry of Health Community Development Gender Elderly and Children

\section{William H Dow}

University of California Berkeley School of Public Health

\section{Nicholas $\mathrm{P}$ Jewell}

London School of Hygiene and Tropical Medicine Department of Medical Statistics

\section{Sandra I McCoy}

University of California Berkeley School of Public Health

\section{Study protocol}

Keywords: HIV, Tanzania, implementation-effectiveness hybrid design, cash transfer, ART adherence, cluster randomized trial

Posted Date: March 12th, 2020

DOl: https://doi.org/10.21203/rs.3.rs-16979/v1

License: (c) (i) This work is licensed under a Creative Commons Attribution 4.0 International License. Read Full License

Version of Record: A version of this preprint was published at Trials on November 23rd, 2020. See the published version at https://doi.org/10.1186/s13063-020-04899-7. 
TITLE: Optimizing the Efficiency and Implementation of Cash Transfers to Improve Adherence to Antiretroviral Therapy: A Cluster Randomized Controlled Trial (Study Protocol)

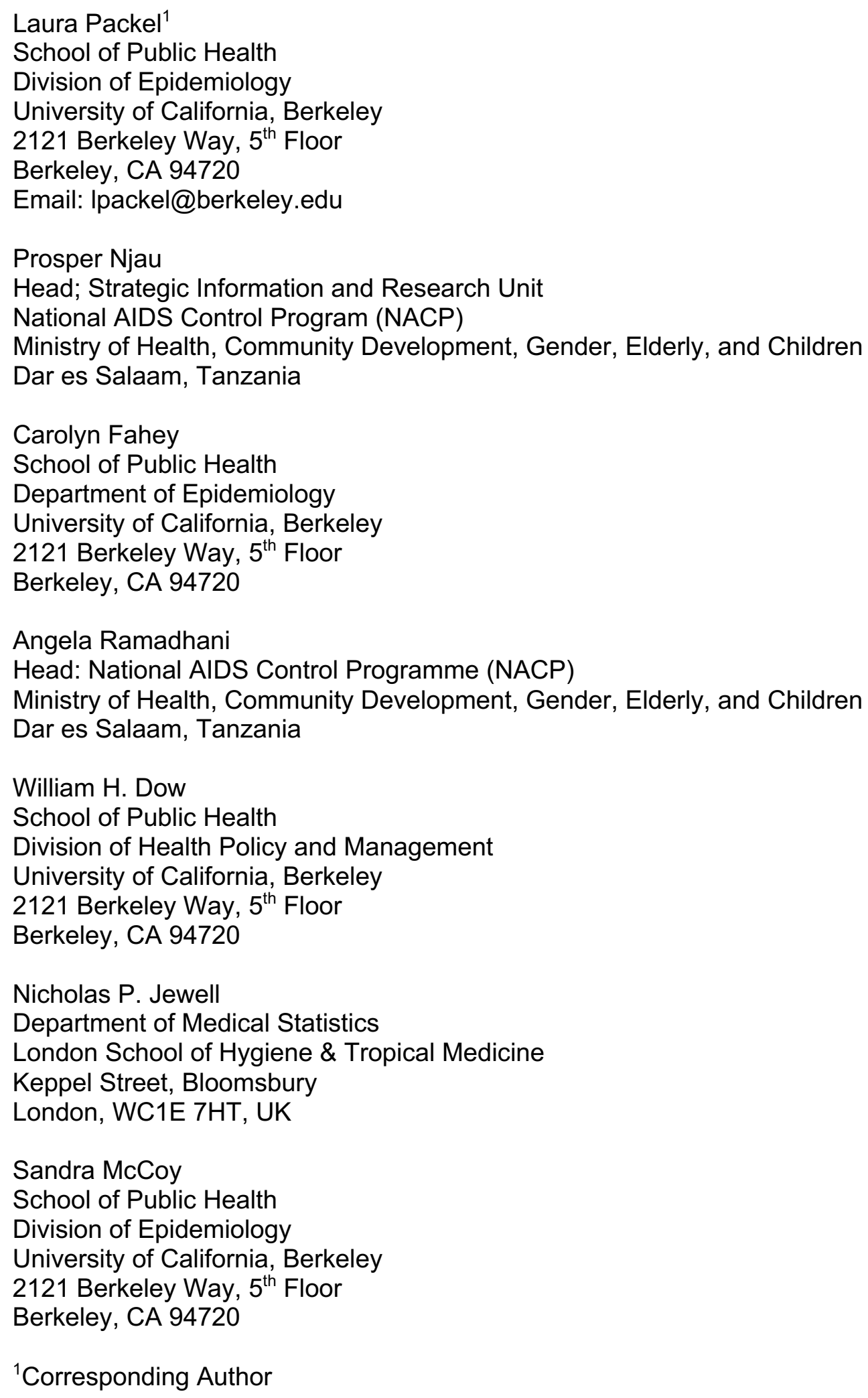




\begin{abstract}
Background: Antiretroviral therapy (ART) for HIV, taken daily, is an effective strategy to clinically suppress the virus, providing the dual benefit of improved survival and vastly decreasing the risk of transmission. However, this highly effective intervention has not yet reached all who could benefit. Cash transfers are increasingly recognized as an effective strategy to motivate behavior change and improve outcomes along the HIV care continuum, including engagement in HIV care and adherence to ART. Despite a growing evidence base and strong theoretical foundation for the cash transfer approach, key questions remain. Specifically, gaps exist in understanding: 1 ) whether short-term cash transfers can increase viral suppression in larger, effectiveness studies; and 2) an implementation model or set of best practices that facilitate scale up and enhance sustainability. To address these questions and begin to bridge the "know-do gap" with respect to cash transfers, our team is employing an implementation science approach to iterative development of an incentive-based implementation strategy to promote ART uptake and adherence among people living with HIV (PLHIV) in the Lake Zone Region, Tanzania.

Methods: We will conduct a type I hybrid implementation - effectiveness trial to test the effectiveness of a cash transfer intervention on the outcome of HIV viral suppression, and concurrently examine the potential for real-world implementation with an mHealth system through measurement of implementation science constructs. Specifically, our team will expand the intervention to 32 clinics and enroll 1,984 PLHIV to (a) evaluate its effectiveness by conducting a cluster randomized controlled trial with clinics as the unit of randomization and 12month viral suppression as the primary outcome - a key indicator for monitoring progress towards HIV epidemic control; and (b) evaluate the implementation challenges and successes by measuring implementation outcomes at multiple levels (patient, provider, clinic).
\end{abstract}

Discussion: This trial will provide evidence about not only the real-world effectiveness of cash transfers for retention in HIV care and viral suppression, but also on the implementation challenges and successes that will facilitate or hinder wider scale-up within Tanzania and beyond.

Trial Registration: Clinicaltrails.gov

Registration Number: NCT04201353

Date of Registration: December $17^{\text {th }}, 2019$

URL for Trial Registration: https://clinicaltrials.gov/ct2/show/NCT04201353

\title{
Contributions to the Literature
}

- Adherence to HIV antiretroviral therapy and resulting viral suppression are widely known as critical for ending the HIV epidemic;

- Effective implementation strategies to ensure that people living with HIV have viral suppression are needed; cash transfers are one such strategy;

- This study protocol describes a cluster randomized controlled trial with an implementation-effectiveness hybrid design to optimize a cash transfer implementation strategy for HIV control in real-world settings.

Keywords: HIV, Tanzania, implementation-effectiveness hybrid design, cash transfer, ART adherence, cluster randomized trial 


\section{Background}

Antiretroviral therapy (ART), taken every day as prescribed, is an effective strategy to clinically suppress the HIV virus, providing the dual benefit of improved health and vastly decreasing or eliminating the risk of onward transmission. Despite the robust evidence, this highly effective intervention has not yet reached all who could benefit. For example, in Tanzania, 1.6 million people are living with HIV, and 72,000 new infections occur yearly. Of those living with HIV, only $62 \%$ are virally suppressed, and of those who are currently on HIV treatment and who have viral load testing results, $87 \%$ are virally suppressed $(1,2)$ - falling short of the 95-95-95 goals that UNAIDS has set for 2030 for which $95 \%$ of those on HIV treatment should be virally suppressed (2). To reach these goals, new and effective implementation strategies are needed that can translate evidence into widespread practice and thereby bring sustained HIV treatment for viral suppression to all people living with HIV (PLHIV).

Conditional economic incentives are increasingly recognized as one such implementation strategy: these programs typically provide cash (or other incentives) to people who meet a particular condition, for example testing for HIV, returning for HIV test results, or testing negative for sexually transmitted infections, thus motivating certain behaviors that result in improved health. Conditional economic incentive programs in the context of improving HIV outcomes have thus far been short term, and primarily implemented in the context of research settings, differentiating them from social protection cash transfer programs that are typically longer-term and funded by the government. There is now a substantial evidence base demonstrating the effectiveness of cash transfer programs in improving outcomes along the HIV care continuum in low resource settings in a research context (2-19). Despite this strong evidence, few of these cash transfer programs to improve HIV outcomes have been scaled, and gaps exist in understanding the long-term impacts of these programs.

To address these questions and begin to bridge the "know-do gap" with respect to ART adherence and viral suppression, we are employing an implementation science approach to the development of a cash incentive program to promote ART adherence among PLHIV in the Lake Zone, Tanzania. We draw on two implementation science frameworks to answer a series of interrelated research questions that would be relevant to future scale-up and longer-term sustainability of a cash transfer intervention. We use Proctor's framework (20) to define implementation constructs of interest with respect to the users, stakeholders, and context of the intervention. In addition, we use an adapted version of the multiphase optimization strategy (MOST) framework (21) to guide the process of optimizing and evaluating the intervention itself. Given a multicomponent intervention, the 3-stage MOST framework (preparation, optimization and evaluation) isolates the effective component(s) of that intervention based on pre-specified optimization criteria and an efficient study design that obviates the need to conduct large, lengthy trials. Then, the optimized intervention is evaluated for effectiveness in a subsequent RCT in a real-world setting. MOST is adaptable to various trial designs provided the key features of optimization and resource management are present.

Guided by these frameworks, our team has designed and conducted a set of iterative experiments to: determine efficacy of the incentive-based intervention, optimize the intervention for a real-world clinical setting, assess the feasibility and acceptability of the intervention package for scale up and sustainability, and finally, test the optimized intervention package for effectiveness on a large scale across multiple care and treatment facilities in Tanzania. Together, this set of trials (efficacy - optimization - effectiveness) will generate an evidence base for the most effective, incentive-based implementation strategy for the clinically proven 
intervention of ART adherence. This paper describes the protocol for the large-scale hybrid effectiveness-implementation (22) science trial.

Results from the first two randomized trials conducted by our team (efficacy and optimization) demonstrated that cash transfers conditional on visit attendance have the potential to improve ART adherence and retention in care among PLHIV in Tanzania. In the first study (efficacy), our team randomized 800 food insecure PLHIV who recently started ART at three clinics to the standard of care or 6 months of cash or food transfers, conditional on visit attendance (15). After 6 months of the intervention, we found that short-term cash transfers were superior to the standard of care on all indicators of adherence and retention, including the medication possession ratio (MPR), a pharmacy-based measure of adherence associated with viral suppression (23-26), appointment attendance, and loss to follow-up (27). After 12 months, 6 months after the intervention ended, the cash group remained more likely to be in care than the standard of care group and had superior appointment attendance. Furthermore, cash transfers were safe to administer $(28,29)$ and compared to food baskets, were equal or superior to food support on all outcomes, were cheaper and easier to monitor, and were preferred by patients. Analysis of individual motivation found that cash transfers do not undermine intrinsic motivation to adhere to HIV treatment; indeed, intrinsic motivation increased over time as PLHIV experienced the benefits of treatment (30). Qualitative research revealed that money received as part of the intervention was largely being spent on food, school fees, and investment in assets and small businesses.

Building on these proof-of-concept results, in a second trial (optimization) we further drew on constructs from implementation science (31), including Proctor's outcomes and the MOST framework, to refine and optimize the intervention among 530 PLHIV initiating ART at four clinics. This second study (completed in August 2019, ClinicalTrials.gov: NCT03351556) evaluated two cash transfer sizes $(\sim 5$ and $\sim \$ 10)$ conditional on visit attendance, and for the larger amount, confirmed the first trial's results using viral suppression as the outcome at 6months (risk difference vs. control=13.1 percentage points, $95 \% \mathrm{Cl}$ : $4.5-21.5$ ). The study also assessed whether a clinic-based mHealth system could streamline intervention implementation; the system was designed to identify patients biometrically (using fingerprints) and to automatically transfer cash via mobile money to eligible patients upon scanning in for their visit (32).

Armed with evidence on the intervention's efficacy and an optimized implementation strategy using the larger cash amount and mHealth system, we will now conduct the third study, a Type I hybrid implementation - effectiveness trial. This study will test the effectiveness of the cash transfer intervention on the outcome of viral suppression six months after the cash program ends, and concurrently examine the potential for real-world implementation through measurement of implementation science constructs (22). Specifically, our team will expand the intervention to 32 clinics to: (a) evaluate its effectiveness by conducting a cluster randomized controlled trial with clinics as the unit of randomization and 12-month viral suppression as the primary outcome - a key indicator used in monitoring global progress towards HIV epidemic control; and (b) evaluate the challenges and successes by measuring implementation outcomes at multiple levels (patient, provider, clinic) following Proctor's framework and constructs. This is the next, logical stage of this research as we build the evidence base for the eventual adoption of a streamlined version of this intervention at scale.

\section{Methods}

\section{Design}


The study has two primary objectives:

1. Impact Evaluation: Evaluate the effectiveness of the 6-month cash transfer program at improving the proportion of PLHIV retained on ART and with suppressed viral load $(<1000$ copies/ml) at 12 months after starting ART.

The primary objective of this study is to evaluate the effectiveness of the cash transfer program using 22,500 TZS/month (as determined by Phase I) at improving the proportion of PLHIV retained on ART and with suppressed viral load at 12 months after starting ART. Using a cluster randomized control trial, with our sample size of 32 clinics and 1,984 participants (62 participants per clinic), we will have $80 \%$ power to detect a risk difference (RD) of 11 percentage points in viral suppression at 12 months between the participants attending intervention and control clinics.

The secondary outcomes of the impact evaluation are:

- Viral suppression (<1000 copies/ml) at 6 months;

- Retention on ART at 6 and 12 months;

- The proportion virally suppressed of those retained on ART at 6 and 12 months; and

- Appointment attendance, the proportion of scheduled visits that were completed during the $0-6$ and $0-12$ month periods.

2. Implementation Study: Understand implementation successes and challenges through measurement of implementation outcomes, and use lessons learned to inform wider adoption of cash transfer programs for PLHIV.

Although evidence from our previous studies demonstrate preliminary effectiveness of cash transfers on HIV-related outcomes, we do not yet know the optimal strategy for implementation of this type of system at a larger scale and outside of a research setting. Thus, in anticipation of the potential adoption of the program by the Ministry of Health or others, a primary objective of this study is to gather information related to successful implementation practices and challenges needing attention before scaling.

To address these aims, we will conduct a Type I hybrid implementation-effectiveness study using a two-arm, cluster randomized controlled trial (Objective \#1, "impact evaluation") with the health facility as the unit of randomization (see Figure 1). To measure implementation outcomes (Objective \#2, "implementation study") following Proctor's framework (33), quantitative and qualitative interviews will be conducted with clinic staff (pharmacy staff, registration staff, clinicians), clinic management, and patients at participating sites. Results will be used to assess heterogeneity in implementation practices and thus inform optimization for potential scale-up. The study will be conducted at 32 HIV primary care clinics in the following Lake Zone regions in Tanzania: Shinyanga, Mwanza, Kagera, and Geita.

[Figure 1 Here] 


\section{Theoretical Framework}

The use of incentives for ART treatment adherence is supported by several theories, including Self Determination Theory, which describes engagement in an activity because of an external reward like a cash transfer.(34) In addition, microeconomic theory posits that people acquire more of a less costly good, and less of a more expensive one (35) and that individuals often have high discount rates or "present-biased preferences," placing disproportionate weight on the present while largely ignoring the future. $(36,37)$ An implication is that when a behavior, like attending an HIV care visit, has small immediate costs and large delayed benefits, a small immediate incentive may counteract the present costs and tip the balance towards the positive behavior. $(36,38)$ The use of incentives for behavior change is also supported by behavioral economic theory, which incorporates constructs from psychology to account for the predicable irrationalities, heuristics, and biases of human behavior.(39) For example, "nudges" or shortterm, small incentives can change behavior $(40,41)$ and create new habits $(42)$ - a goal of the short-term cash transfer intervention.

The implementation science portion of the hybrid trial is guided largely by two frameworks: Proctor's implementation science framework, which explores implementation successes and challenges at multiple levels, including the patient, provider and clinic. We also draw from the MOST framework, designed to accelerate the development and rollout of effective, optimized interventions at the lowest cost possible. The study design follows closely the typology of hybrid designs outlined by Curran et al (22) (Type I in our case), for which the primary question is related to whether the intervention will work in real-world clinical settings (stage 3 of the MOST framework); and the secondary question relates to the potential barriers and facilitators of widespread adoption of the intervention (Proctor's framework).

\section{Clinic Recruitment}

We will engage Regional Medical Officers (RMOs) in Shinyanga, Geita, Mwanza and Kagera Regions, Tanzania to create a sampling frame of eligible study facilities within each region. Initial eligibility criteria for clinics is as follows:

- Currently use an electronic medical records database;

- Average of at least 65 new ART initiates per quarter in 2019, with no fewer than 35 ART initiates in any single quarter in 2019;

- Within $100 \mathrm{~km}$ driving distance of a city center (either Bukoba, Mwanza, Geita, Kahama, or Shinyanga cities); and

- A minimum of $15 \mathrm{~km}$ from another study clinic.

The HPON team will approach and enroll eligible selected sites in a randomly assigned order (1 to 10 within each region) until the target enrollment is reached, with the goal of having 32 sites enroll in the study.

\section{Study Arms}

\section{Control Arm}

Participants in the control arm will receive the standard of care for PLHIV per the National Guidelines in Tanzania (43), as delivered by the participating health facilities. In addition to the standard of care, the mHealth system developed and utilized in the previous (optimization) trial will be implemented in all study clinics (intervention and control). This system was successfully used in the clinics to enroll study participants, track patient appointment attendance, and disburse cash transfer payments for those eligible using automated mobile money transfers 
(32). We will adapt this mHealth system for the current study and implement it in all study facilities (intervention and control) for enrolling patients, tracking all patient visits, sending appointment reminders (regardless of eligibility for the cash transfer or intervention group), and sending cash transfers via mobile banking (cash transfer group only; consistent with procedures used in our previous studies). This strategy is consistent with the Ministry of Health goal of using mHealth strategies for patient care management in Tanzania, as incorporated into the National Strategy for HIV Care (44).

\section{Intervention Arm}

In addition to the mHealth system as described above, participants in the intervention arm will have the opportunity to receive up to 6 consecutive monthly cash transfers of 22,500 TSH $(\sim 10)$ each, conditional on visit attendance with the HIV care provider. Cash transfers will be given once monthly for up to 6 months, spaced $\geq 25$ days apart (consistent with National Guidelines for monthly or bimonthly visits (43)) and are conditional on visit attendance. This means that the cash transfer is only given when the patient visits the clinic for their routine appointment, regardless of whether the visit is earlier or later than the scheduled appointment (but no earlier than 25 days since the last transfer). Upon the monthly visits when a patient checks in for their appointment (via fingerprint or entry of clinic ID), the cash transfer will be automatically distributed through the mHealth system. Transfer amounts are exclusive of transaction fees $(<\$ 1)$, which will also be included in the transfer. Thus, the intended amount is transferred to the patient upon withdrawal.

If a participant does not have a mobile money account: 1) s/he can sign up and return to the clinic on a different day to link this account to the mHealth system, or 2) s/he may ask a trusted friend or relative to receive the transfer. Mobile money is a secure and convenient way for financial transactions, as mobile money kiosks are ubiquitous in the study regions and mobile phone ownership in Tanzania is high and approaching levels in the U.S. (75\% in 2018 and rapidly increasing (45)). For participants who do not have mobile banking, wherever possible we will make referrals to kiosks where they can sign up for the service, if desired. In the most recent study, we found that $88 \%$ of participants had access to a mobile phone and $79 \%$ had access to mobile banking.

Rationale for the cash amount and duration Our now-completed study to optimize the intervention found that both cash transfer amounts (10,000 TSH and 22,500 TSH per month) significantly improved 6-month viral suppression versus the standard of care. At 6 months, we found a positive trend between incentive size and HIV viral suppression (OR=1.10 per $2500 \mathrm{TZS}, 95 \% \mathrm{Cl}: 1.03$ to 1.17). Further, we found that both cash amounts significantly improved HIV viral suppression over the comparison group [10,000 TSH: $83 \%$ vs. $73 \%$, risk difference $(\mathrm{RD})=10.2,95 \% \mathrm{Cl}: 1.6-18.8 ; 22,500 \mathrm{TSH}: 85 \%$ vs. $73 \%, \mathrm{RD}=12.1,95 \% \mathrm{Cl}: 3.6-20.6]$; there was no significant difference between incentive groups ( $R D=1.9,95 \% \mathrm{Cl}:-5.8$ to 9.7 ). To determine the final cash transfer size for the current study, we followed our pre-registered analysis plan (at AsPredicted) as well as several additional analyses including: a cost analysis, discussions with local stakeholders and co-investigators, assessment of budgetary trade-offs associated with a smaller cash transfer versus larger study size, and consideration about the benefits and weaknesses of various cash amounts (including power considerations and the ethics of engaging in a statistically under-powered study). Based on these data, we determined that the effectiveness evaluation will include a cash transfer amount of 22,500 TSH per month for 6 months.

The intervention is intended to fit within the differentiated model of care in Tanzania, in which patients starting ART are scheduled monthly clinic visits until the first viral load test at 6 months 
after ART initiation. At that time, "stable" patients (those who have attained viral suppression) are given two/three-month refill appointments and/or the opportunity to send a substitute for ART refills or to obtain refills at community-based locations). However, at 6 months, unstable patients (those without viral suppression) continue monthly visits, with enhanced adherence interventions or change of regimen, depending on clinical, immunological, and virological criteria, until they are determined to have stabilized. Thus, the intervention is intended to support patients during the vulnerable first 6 months of treatment before the 6-month decision point for access to a less intensive clinic schedule.

\section{Randomization and Masking}

Once facilities are selected for the study and have agreed to participate, we will randomize 32 facilities to either the SOC or the cash transfer intervention group at UC Berkeley using the cvcrand package (cvrall command) in R statistical software (46). To ensure that the arms are balanced on important covariates, and to mitigate the possibility of ineffective randomization due to the small numbers of clinics in the study, we will use a constrained randomization process (47). This process essentially pre-determines a set of acceptable allocations into intervention and control clusters based on covariates of relevance, and then randomly selects an allocation from the list of acceptable allocations (47). Specifically, based on our experience in phase 1, we will include the following covariates in the constrained randomization process: geographic region (Geita, Kagera, Mwanza or Shinyanga), facility level (hospital, health center or dispensary), driving distance to a major city $(\mathrm{km})$, proximity to a major road $(<5 \mathrm{~km})$, and average number of ART initiates per quarter. We will stratify on geographic region. The 32 sites will be randomized 100,000 times and we will select the unique schemes as the randomization space. Those iterations with an 12 balance score less than the $q=0.1$ cutoff will be retained. Among those remaining iterations where there was little to no imbalance detected, we will check for validity of the constrained randomization (e.g., no deterministic allocation of clusters into arms) and ensure that there are sufficient constrained randomizations from which to randomly select a single randomization scheme.

Facility staff will not be blinded to intervention assignment. However, other clinic staff will not be informed that there are intervention and control clinics in the study, and clinical staff trainings for intervention clinics and control clinics will be conducted separately. In addition, participants will not be told during the consent process that as part of the study there are intervention and control clinics. The rationale for this is to prevent patients transferring from intervention to control clinics if they find out that there are some clinics offering cash transfers to new ART clients. Although this is not expected to be a significant problem based on previous studies of this approach, large numbers of transferring patients could compromise the integrity of the study and will create an undue burden for facility staff at intervention clinics.

\section{Recruitment of Participants}

Impact Evaluation: Recruitment of individuals for participation in the impact portion of the study will take place within the enrolled clinics. All patients at study clinics who meet the following inclusion criteria will be offered the opportunity to participate in the study:

- Greater than or equal to 18 years of age

- Living with HIV infection

- Initiated on ART (for the first time) less than or equal to 30 days prior to enrollment in the study

- Have access to a mobile phone (ownership, shared ownership, or access to a trusted person's phone)

- Do not intend to transfer to a different facility for HIV care within the next 12 months. 
Eligible PLHIV at both intervention and standard of care sites will be automatically identified by the mHealth system upon registration in the system at a routine visit. An automatic prompt will direct clinic registration staff (who will manage the process) to the appropriate consents and forms in the mHealth system, including permission to access patient data as part of the study. If the patient consents, s/he will automatically be assigned a study ID. The study will use noncompetitive enrollment so that we can achieve approximately equal numbers of study participants per site, with a goal of 62 per site. Sites that reach this goal early will discontinue enrollment.

Implementation Study: Recruitment for participation in the implementation science portion of the study for clinic staff will take place in the intervention clinics once all participants have reached their 6-month post-ART timepoint and the cash transfer period (of 6 months) has ended. We will purposefully select staff from the all study clinics such that our interviews include all levels of staff impacted by the changes in the clinic as related to implementation of the intervention (e.g., nurses, registration staff, physicians, In-Charges, pharmacists). Similarly, patients who have consented to and enrolled in the study will be purposefully sampled to assess their experiences with the cash transfer program and the mHealth system after their completion of cash transfer eligibility at 6 months. Specifically, we will interview patients who: a) received all six cash transfers; b) received three or fewer of the six possible transfers; $c$ ) enrolled in mobile money as a result of the study; d) were virally suppressed at 6 months; e) were not virally suppressed at 6 months in addition to ensuring representation of both men and women and a range of ages.

\section{Outcomes and Data Collection}

Primary outcome measure (impact evaluation, Objective \#1): The primary endpoint is viral suppression at 12 months, defined as the proportion of PLHIV retained in HIV primary care and with suppressed HIV viral load 12 months after starting ART. The primary outcome is expressed as a binary variable, defined as PLHIV who are on ART and with sufficient HIV viral suppression (<1000 copies/ml, WHO's threshold for virologic failure in LMICs(48)) versus not on ART or viral failure ( $\geq 1000$ copies $/ \mathrm{ml}$ ). This outcome definition reflects global "treatment as prevention" strategies including the UNAIDS 95-95-95 targets, which aim for at least 95\% of PLHIV to be on ART and $95 \%$ of those on ART virally suppressed. Patients considered not on ART include those who died, stopped ART and/or disengaged from care, or have not apparently received ARVs for $\geq 28$ days since their last missed pharmacy pick-up [i.e., are lost to follow-up (LTFU)] following PEPFAR Monitoring, Evaluation, and Reporting Indicator Reference Guide Version 2.3 for current ART(49).

Secondary outcome measures (impact evaluation, Objective \#1):

- Viral suppression (<1000 copies/ml) at 6 months;

- Retention on ART at 6 and 12 months;

- The proportion virally suppressed of those retained on ART at 6 and 12 months; and

- Appointment attendance, the proportion of scheduled visits that were completed during the $0-6$ and $0-12$ month periods.

Primary outcome measures (implementation study, Objective \#2): Implementation outcomes assessed through in-depth interviews and surveys with patients and clinical staff: Adoption, acceptability, penetration, fidelity, feasibility, appropriateness, sustainability; plus usability (Table $1)$. 
[Table 1 Here]

Data Collection: Data collection will include the following:

- Medical record review for the primary outcome, viral suppression, at 6 and 12 months

- Surveys with patients at intervention and control clinics at baseline and 6 and 12 months $(n=1,984)$

- In-depth interviews with patients in the intervention clinics at 6 months $(n=30)$

- Surveys with clinical staff at intervention and control clinics at 6 months $(n=110)$

- In-depth interviews with clinical staff at intervention and control clinics at 6 months $(n=40)$.

We linked each data collection activity for the implementation study to implementation outcomes in the Proctor et al. framework (Table 1). (33) We will analyze data from structured surveys and in-depth interviews to examine the implementation constructs of acceptability, penetration, sustainability, appropriateness, adoption, feasibility, and fidelity. We will use validated surveys to assess the usability of the mHealth system from both the clinic staff and patient perspectives, including the System Usability Scale (50) and the Health Information Technology Usability Evaluation Scale (51).

Participant Tracing - Impact Evaluation: As retention in care is included as part of the definition of the primary outcome of this study (i.e., those not retained on ART are included in the denominator of the primary outcome of viral suppression), no additional retention activities will be implemented other than the routine procedures already in place and led by clinical staff. According to national guidelines, patients who miss a scheduled appointment are tracked in the community by a system of home-based care providers (HBCs); this system will remain in place during the study. Research staff will not be involved in tracing participants at any study facility during the 12-month follow-up of each participant. At endline only (12 months), HPON research assistants will enhance these procedures with additional robust tracing using 'gold-standard' tracing methods (at least 3 tracing attempts using multiple methods), procedures to investigate all potentially LTFU patients, confirm 'silent transfers' (those who transfer to new facilities without notification of the prior facility) and deaths, and refer patients to health facilities where missing plasma specimens can be collected by health facility staff for viral load quantification.

\section{Statistical Analysis}

Power Calculation: The sample size was determined for the impact study to evaluate the effectiveness of the cash transfer program at improving the proportion of PLHIV with suppressed viral load (<1000 copies $/ \mathrm{ml}$ ) at 12 months. We used estimates of viral suppression at 12 months and ICC $=0.05$, estimated from a previous cluster randomized study of HIV treatment adherence that we conducted among HIV-infected pregnant women in Shinyanga Region, an ICC consistent with previous studies.(52) Using site-adjusted data about viral suppression at 6 months from Phase I (75.2\% in the control group), along with estimates of viral suppression decline between 6 and 12 months, we estimate that viral suppression among adults at 12 months in the comparison sites will (conservatively) be $70 \%$. With an ICC of $0.05,32$ facilities, $80 \%$ power, and 56 PLHIV per clinic $(n=1,792)$, the minimum detectable effect is 11 percentage points as an absolute reduction in the proportion experiencing viral suppression. In the second trial (optimization) we achieved a 12.6 percentage point difference with the 22,500 TSH cash transfer at 6 months, so we believe this effect size is achievable. In addition, if viral 
suppression is lower than expected in the comparison arm, and the effect of the intervention remains stable, power will increase. We will inflate the target sample size by $10 \%$ to $n=1,984$ (62 participants per clinic across 32 clinics) to account for unexpected issues or significant differences in the enrollment between clinics.

Data Analysis - Impact Study: We will conduct an intent-to-treat analysis to determine the effect of the intervention on 12-month viral suppression (the primary outcome). We will conduct a cluster-based permutation test on the individual-level outcome data, which accounts for clustering within clinic. The effect estimate of interest is the risk difference of viral suppression among PLHIV attending intervention versus comparison facilities. We will pre-register our analysis plan at AsPredicted.

We will conduct several secondary analyses of the primary outcome, although we are not powered for these analyses. We will examine heterogeneity in the primary analysis by facility and patient characteristics. We will also conduct a treatment-on-the-treated analysis to isolate the impact of incentive delivery (53-55).

Using the same methods as for the primary analysis, we will also assess effects on the following key secondary outcomes:

- Viral suppression (<1000 copies/ml) at 6 months;

- Retention on ART at 6 and 12 months;

- The proportion virally suppressed of those retained on ART at 6 and 12 months; and

- Appointment attendance, the proportion of scheduled visits that were completed during the $0-6$ and $0-12$ month periods.

We will additionally assess effects on the following survey-based outcomes at 6 and 12 months:

- Food security

- Mental health (anxiety, depression)

- Hopefulness

- IPV

- Self-efficacy

- Participation in the labor force / functional status

- Other indicators of household welfare (e.g., investment in small businesses)

Data Analysis - Implementation study: Following each in-depth interview, the interviewer will memo then debrief with the study team to discuss emergent themes. Debriefings will be held weekly with interviewers and the PI to ensure consistency and quality of data collection. Audiorecordings from IDIs will be verbatim transcribed and translated into English. Data analysis will follow an open-coding approach $(56,57)$ and will be based on research questions, study aims, and Proctor's implementation constructs. An initial coding framework will include a list of predefined analytical terms relating to Proctor's constructs; however, data analysis will be iterative, allowing new themes to emerge throughout the analysis process(58). Data will be coded independently by two members of the research team using Dedoose qualitative coding software. Concepts will be grouped into categories, and main themes will be extracted and summarized in an analytic theme matrix.

Implementation outcome data from structured surveys with clinical staff will be analyzed descriptively using both STATA and Dedoose. We will synthesize quantitative and qualitative data to produce a comprehensive set of implementation outcome indicators. We will then use the implementation indicators to assess variation in how the program was implemented across 
the intervention clinics, for example, exploring levels of penetration and fidelity of the mHealth system, and explore variation in effectiveness using a sensitivity analysis. Note that we are not powered to show statistical differences in viral suppression by variation in implementation outcomes.

\section{Discussion}

Achieving global goals for HIV epidemic control will necessitate new implementation strategies to identify PLHIV, link them to high-quality HIV care and treatment, and retain them on lifelong ART. Cash transfers have emerged as a promising strategy worthy of consideration as part of a comprehensive approach to HIV prevention and care. We have used an iterative process grounded in implementation science approaches (MOST, Proctor) to demonstrate efficacy of cash transfers for ART adherence (trial 1), optimize the cash intervention with respect to cash amount (trial 2), and now, in our third trial, we will evaluate effectiveness of cash transfers on viral suppression with a contemporaneous assessment of implementation outcomes with the goal of preparing the intervention for scale in the complex context of LMIC health systems. The results from this type I hybrid implementation - effectiveness trial will provide much-needed results on not only the real-world effectiveness of cash transfers for ART adherence and viral suppression, but also on the implementation challenges and successes that will facilitate or hinder wider scale-up within Tanzania and beyond.

The hybrid approach (effectiveness/IS) will permit: a) a contribution to what is known about the use of short-term cash transfers for PLHIV in a real-world setting in sub-Saharan Africa, including their long-term effects on viral suppression; and b) an understanding of how to optimize implementation approaches, and thus, provide guidance for policy makers in facilitating successful scale-up. These results have the potential to provide the evidence needed to show that short-term assistance confers lasting benefits for those starting treatment, providing needed support as they begin to feel the beneficial effects of ART. Furthermore, the study is based in the Lake Zone region of Tanzania, a region that faces challenges common throughout subSaharan Africa: a shortage of skilled personnel, poverty and food insecurity, and pervasive challenges with retention in care. For these reasons, our results are likely to have a high level of external validity and policy relevance in sub-Saharan Africa.

We have intentionally designed the hybrid trial to be largely self-sufficient - that is, clinical staff have the majority of interaction with the research participants, with support from research staff initially, and then only as needed. This approach comes with some tradeoffs and limitations. First, there is the strong possibility that implementation of the intervention may differ by site. While implementation differences have the potential to dilute the effect of the cash program, for an effectiveness trial in a real-world setting, such variation will provide essential knowledge that will allow best practices and lessons learned to emerge and guide policy makers in bringing the program to scale. Second, patients attending control clinics may hear about the intervention, and transfer out of control clinics an into intervention clinics. To mitigate this possibility (though this was not an issue in first two trials), we have included a minimum geographic distance between clinics to minimize spillover effects and prevent contamination of comparison communities. In addition, as large numbers of transferring patients would compromise the integrity of the study and will create an undue burden for facility staff at intervention clinics, we will not reveal that there are intervention clinics where patients will receive cash transfers as part of the study to patients enrolling in the study at control clinics. Third, there is substantial variability over time and across clinics in the number of new ART initiates, and this variability may potentially impact the rate at which we are able to enroll patients into the study. 
The results of this hybrid implementation-effectiveness trial will guide policymakers in Tanzania and elsewhere in the Region about whether this intervention should (or should not) be considered in national HIVIAIDS programs, and if it should, how implementation should proceed to optimize the effectiveness of the intervention in clinics across the country. Consequently, this effectiveness trial has been designed with an eye towards real-world, future scale-up, such that the procedures we propose to follow align as closely as possible with National policy and consistent with what clinics would and could do outside of a research study setting. 


\section{List of Abbreviations}

ART ............Antiretroviral Therapy

PLHIV..........People Living with HIVIAIDS

TZS............Tanzanian Shillings

LTFU.............Loss to follow-up

RA.............Research Assistant

CTC............ Care and Treatment Clinic (HIV)

IS..............Implementation Science

HPON ......... Health for a Prosperous Nation

MOST..........Multiphase Optimization Strategy

\section{Declarations}

Ethics approval and consent to participate: We have received approval from the University of California Committee for Protection of Human Subjects and the Tanzanian National Medical Research Institute.

Consent for publication: Not applicable

Availability of data and materials: Not applicable

Competing interests: The authors declare that they have no competing interests.

Funding: This work was supported by grant R01MH112432 from the National Institute of Mental Health. The content is solely the responsibility of the authors and does not necessarily represent the official views of the National Institute of Mental Health or the National Institutes of Health.

Author's contributions: LP wrote the manuscript and provided input and expertise specific to the implementation science section of the study. SM and PN conceived of and designed the study and provided guidance on all aspects of the protocol. LP and CF provided input on all aspects of study design. CF conducted the site selection and randomization. PN, NJ, WD, and AR provided expert guidance on study implementation, power calculations, randomization and statistical analysis. All authors read and approved the final manuscript.

Acknowledgements: We acknowledge the contributions of Dr. Nancy Padian and Dr. Stefano Bertozzi to earlier phases of this research portfolio. We are grateful for the excellent work of our technology partner, Rasello, to our viral load testing partner Management and Development for Health, and to the Regional Medical Officers and Regional Health Management Teams in Shinyanga, Geita, Mwanza, and Kagera for their thoughtful guidance on study implementation. In particular, we are grateful to Dr. Rashid Mfaume, the Shinyanga Regional Medical Officer, and Dr. Ntuli Kapologwe, Director of Health, Social Welfare \& Nutrition Services in the President's Office, Regional Administration and Local Government (PO-RALG), without whom these studies could not have been possible.

Author's information (optional) 


\section{$\underline{\text { References }}$}

1. Avert. Global information and education on HIV and AIDS [Internet]. [cited 2019 Dec 1]. Available from: https://www.avert.org/professionals/hiv-around-world/sub-saharanafrica/overview

2. HIVIAIDS JUNP on, others. Fast-track: ending the AIDS epidemic by 2030. Geneva UNAIDS. 2014;

3. McCoy SI. Improving the Efficiency of HIV Testing With Peer Recruitment, Financial Incentives, and the Involvement of Persons Living with HIV Infection. J Of. 2013;

4. Björkman-Nyqvist M. Evaluating the impact of short term financial incentives on HIV and STI incidence among youth in Lesotho: a randomized trial. In: 7th IAS Conference on HIV Pathogenesis, Treatment, and Prevention. Kuala Lumpur; 2013.

5. Baird SJ. Impact of a cash transfer program for schooling on prevalence of. In: HIV and HSV-2 in. Malawi: a;

6. de Walque D. Incentivising safe sex: a randomised trial of conditional cash transfers for HIV and sexually transmitted infection prevention in rural Tanzania. BMJ Open. 2012;2:000747.

7. Galarraga O. Conditional Economic Incentives to Improve HIV Treatment Adherence: Literature Review and Theoretical Considerations. AIDS Behav. 2013;

8. Solomon S. The Impact of Voucher Incentives on Linkage to Care and ART Initiation among Drug Users. In: Chennai, India in 19th Conference on Retroviruses and Opportunistic Infections. 2012.

9. Haukoos JS. The effect of financial incentives on adherence with outpatient human immunodeficiency virus testing referrals from the emergency department. Acad Emerg Med. 2005;12(7):617-21.

10. Thornton R. The Demand for and Impact of Learning HIV Status: Evidence from a Field Experiment. Am Econ Rev. 2008(98):1829-63.

11. Cluver L. Child-focused state cash transfers and adolescent risk of HIV infection in South Africa: a propensity-score-matched case-control study. Lancet Glob Health. 2013;1(6):362-370.

12. Handa $S$. The government of Kenya's cash transfer program reduces the risk of sexual debut among young people age 15-25. PLoS One. Vol. 9. 2014. 85473 p.

13. Singer AW, Weiser SD, McCoy and SI. Does Food Insecurity Undermine Adherence to Antiretroviral Therapy? Syst Rev AIDS Behav. 2014;

14. Young S. A Review of the Role of Food Insecurity in Adherence to Care and Treatment Among Adult and Pediatric Populations Living with HIV and AIDS. AIDS Behav. 2013; 
15. McCoy SI. Rationale and design of a randomized study of short-term food and cash assistance to improve adherence to antiretroviral therapy among food insecure HIVinfected adults in Tanzania. BMC Infect Dis. 2015;15:490.

16. Buzdugan R, Bautista-Arredondo S, McCoy and SI. The promise of behavioral economics for HIVIAIDS treatment and care. In: 21th International AIDS Conference. Durban: South Africa; 2016.

17. Chaiyachati $\mathrm{KH}$. Interventions to improve adherence to antiretroviral therapy: a rapid systematic review. AIDS. 2014;2:187-204.

18. El-Sadr WM. Effect of Financial Incentives on Linkage to Care and Viral Suppression. In: HPTN 065 in Conference on Retroviruses and Opportunistic Infections. 2015.

19. Galarraga O, Sosa-Rubi SG. Conditional economic incentives to improve HIV prevention and treatment in low-income and middle-income countries. Lancet HIV. 2019 Oct;6(10):e705-14.

20. Proctor E, Silmere H, Raghavan R, Hovmand P, Aarons G, Bunger A, et al. Outcomes for Implementation Research: Conceptual Distinctions, Measurement Challenges, and Research Agenda. Adm Policy Ment Health Ment Health Serv Res. 2011 Mar 1;38(2):6576.

21. Collins LM, Murphy SA, Nair VN, Strecher VJ. A strategy for optimizing and evaluating behavioral interventions. Ann Behav Med. 2005;30(1):65-73.

22. Curran GM, Bauer M, Mittman B, Pyne JM, Stetler C. Effectiveness-implementation Hybrid Designs. Med Care. 2012 Mar;50(3):217-26.

23. McMahon JH. Pharmacy adherence measures to assess adherence to antiretroviral therapy: review of the literature and implications for treatment monitoring. Clinical infectious diseases. Off Publ Infect Dis Soc Am. 2011;52(4):493-506.

24. Messou E. Association between medication possession ratio, virologic failure and drug resistance in HIV-1-infected adults on antiretroviral therapy in Cote d'Ivoire. Journal of acquired immune deficiency syndromes. Vol. 56. 2011. 356-64 p.

25. Goldman JD. Simple adherence assessments to predict virologic failure among HIVinfected adults with discordant immunologic and clinical responses to antiretroviral therapy. AIDS Res Hum Retroviruses. 2008;24(8):1031-5.

26. Hong SY. Medication possession ratio associated with short-term virologic response in individuals initiating antiretroviral therapy in Namibia. PLoS One. Vol. 8. 2013. 56307 p.

27. McCoy SI, Njau PF, Fahey C, Kapologwe N, Kadiyala S, Jewell NP, et al. Cash vs. food assistance to improve adherence to antiretroviral therapy among HIV-infected adults in Tanzania. AIDS Lond Engl. 2017 Mar 27;31(6):815-25.

28. Czaicki NL, Mnyippembe A, Blodgett M, Njau P, McCoy SI. It helps me live, sends my children to school, and feeds me: a qualitative study of how food and cash incentives may 
improve adherence to treatment and care among adults living with HIV in Tanzania. AIDS Care. 2017;29(7):876-884.

29. Kadota J, Fahey C, Njau P, Kapologwe N, Padian NS, Dow W, et al. Conditional food or cash transfers do not increase purchase of temptation goods among adults living with HIV infection in Shinyanga, Tanzania. In 2017.

30. Garcia M and CMTM. The Cash Dividend: The Rise of Cash Transfer Programs. In: SubSaharan Africa, in Directions in Development. Washington, D.C: The World Bank; 2012.

31. Packel L, Fahey C, Njau P, McCoy SI. Implementation Science Using Proctor's Framework and an Adaptation of the Multiphase Optimization Strategy: Optimizing a Financial Incentive Intervention for HIV Treatment Adherence in Tanzania. J Acquir Immune Defic Syndr 1999. 2019 Dec;82 Suppl 3:S332-8.

32. Fahey CA, Mwenda N, Bhattarai PR, Mneney J, Katabaro E, Njau PF, et al. Feasibility and acceptability of a biometric mHealth system for monitoring retention in HIV services and delivering financial incentives to adults initiating antiretroviral therapy in Tanzania. In 2019.

33. Proctor EK, Landsverk J, Aarons G, Chambers D, Glisson C, Mittman B. Implementation research in mental health services: an emerging science with conceptual, methodological, and training challenges. Adm Policy Ment Health Ment Health Serv Res. 2009;36(1):2434.

34. Deci $E$ and RMR. Facilitating optimal motivation and psychological well-being across life's domains. Can Psychol. 2008(49):14-23.

35. Nechyba T, Microeconomics. Mason, Ohio: Cengage Learning; 2010.

36. Loewenstein G, Brennan T, Volpp KG. Asymmetric Paternalism to Improve Health Behaviors. JAMA. 2007 Nov 28;298(20):2415.

37. O'Donoghue T and MR. Doing it now or Later. Am Econ Rev. 1999;89:103-124.

38. Galarraga O. Conditional Economic Incentives to Improve HIV Treatment Adherence: Literature Review and Theoretical Considerations. AIDS Behav. 2013;

39. Tversky A and DK. Judgment under Uncertainty: Heuristics and Biases. Science. 1974;185(4157):1124-31.

40. Codagnone $\mathrm{C}$. The challenges and opportunities of "nudging." J Epidemiol Community Health. 2014;68(10):909-11.

41. Susnstein CR and RHT. Nudge: Improving Decisions About Health, Wealth, and Happiness. 2008. New Haven: Yale University Press;

42. Gardner B. A review and analysis of the use of 'habit' in understanding, predicting and influencing health-related behaviour. Health Psychol Rev. 2015 Aug 7;9(3):277-95.

43. National AIDS Control Programme. National Guidelines for the Management of HIV and AIDS. Tanzania: National AIDS Control Programme; 2017 Oct. Report No.: Sixth Edition. 
44. Ministry of Health and Social Welfare. Tanzania National eHealth Strategy $2012-2018$. Tanzania: Ministry of Health, Community Development, Gender, Elderly and Children, National AIDS Control Programme; 2013.

45. Pew Research Center. INTERNET CONNECTIVITY SEEN AS HAVING POSITIVE IMPACT ON LIFE IN SUB-SAHARAN AFRICA. Pew Research Center; 2018 Oct.

46. R Foundation for Statistical Computing. Vienna, Austria;

47. Moulton L. Covariate-based constrained randomization of group-randomized trials. Clin Trials. 2004;1(3):297-305.

48. World Health Organization, Consolidated guidelines on the use of antiretroviral drugs for treating and preventing HIV infection. 2013.

49. President's Emergency Plan for AIDS Relief. PEPFAR monitoring, evaluation, and reporting indicator reference guide. PEPFAR Washington (DC); 2019.

50. Brooke J. SUS-A quick and dirty usability scale. IN JORDAN, PW, THOMAS, B., WEERDMEESTER, BA \& MCCLELLAND, AL (Eds.) Usability Evaluation in Industry. London, Taylor and Francis; 1996.

51. Schnall R, Cho H, Liu J. Health Information Technology Usability Evaluation Scale (HealthITUES) for Usability Assessment of Mobile Health Technology: Validation Study. JMIR MHealth UHealth. 2018 Jan 5;6(1):e4.

52. Taljaard M, Donner A, Villar J, Wojdyla D, Velazco A, Bataglia V, et al. Intracluster correlation coefficients from the 2005 WHO Global Survey on Maternal and Perinatal Health: implications for implementation research. Paediatr Perinat Epidemiol. 2008 Mar;22(2):117-25.

53. Little RJ, Rubin DB. Causal effects in clinical and epidemiological studies via potential outcomes: concepts and analytical approaches. Annu Rev Public Health. 2000;21:121-45.

54. Greenland S. An introduction To instrumental variables for epidemiologists. Int J Epidemiol. 2000 Dec;29(6):1102.

55. Gertler PJ, Martinez S, Premand P, Rawlings LB, Vermeersch CMJ. Impact Evaluation in Practice, Second Edition. World Bank Publications; 2016. 398 p.

56. Miles MB, Huberman AM, Huberman MA, Huberman M. Qualitative data analysis: An expanded sourcebook. sage; 1994.

57. Ulin PR, Robinson ET, Tolley EE. Qualitative methods in public health: A field guide for applied research. John Wiley \& Sons; 2012.

58. Maxwell JA. Qualitative research design: An interactive approach. Vol. 41. Sage publications; 2012. 


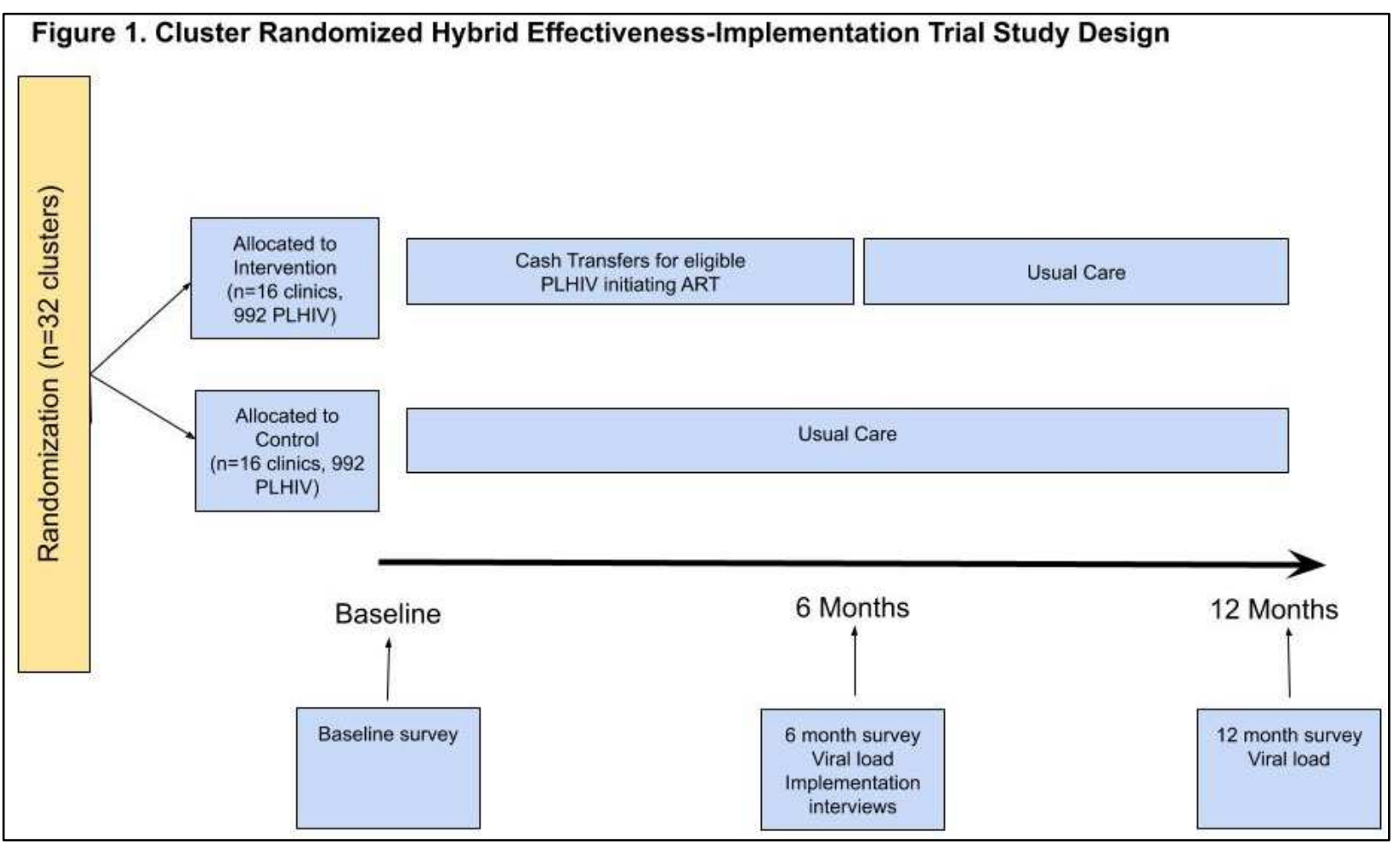

Table 1: Implementation Study: Data Collection Approaches and Proctor's Implementation Science Constructs

\begin{tabular}{|l|l|l|}
\hline Data Collection Approach & $\begin{array}{l}\text { Implementation } \\
\text { Outcomes }\end{array}$ & Outcome Indicators \\
\hline Patient Survey (baseline, 6 months) & $\begin{array}{l}\text { Adoption (mobile } \\
\text { money) } \\
\text { Acceptability }\end{array}$ & $\begin{array}{l}\text { Initiation of use of mobile money; satisfaction with } \\
\text { the program generally and the mHealth system } \\
\text { specifically }\end{array}$ \\
\hline Clinical Staff Survey (6 months) & $\begin{array}{l}\text { Acceptability } \\
\text { Penetration } \\
\text { Fidelity }\end{array}$ & $\begin{array}{l}\text { Staff support and acceptance, level of } \\
\text { institutionalization }\end{array}$ \\
\hline $\begin{array}{l}\text { In-depth interviews with clinic staff } \\
\text { (6 months) }\end{array}$ & $\begin{array}{l}\text { Acceptability } \\
\text { Appropriateness } \\
\text { Sustainability }\end{array}$ & $\begin{array}{l}\text { Staff support and acceptance, staff burden, } \\
\text { practicality and perceived fit }\end{array}$ \\
\hline $\begin{array}{l}\text { In-depth interviews with patients } \\
\text { plus usability survey (6 months) }\end{array}$ & $\begin{array}{l}\text { Acceptability } \\
\text { Appropriateness } \\
\text { Adoption }\end{array}$ & $\begin{array}{l}\text { Patient satisfaction with program and delivery } \\
\text { model, impact on patient, practicality and } \\
\text { perceived fit, adoption of mobile money }\end{array}$ \\
\hline
\end{tabular}


Figures

Figure 1. Cluster Randomized Hybrid Effectiveness-Implementation Trial Study Design

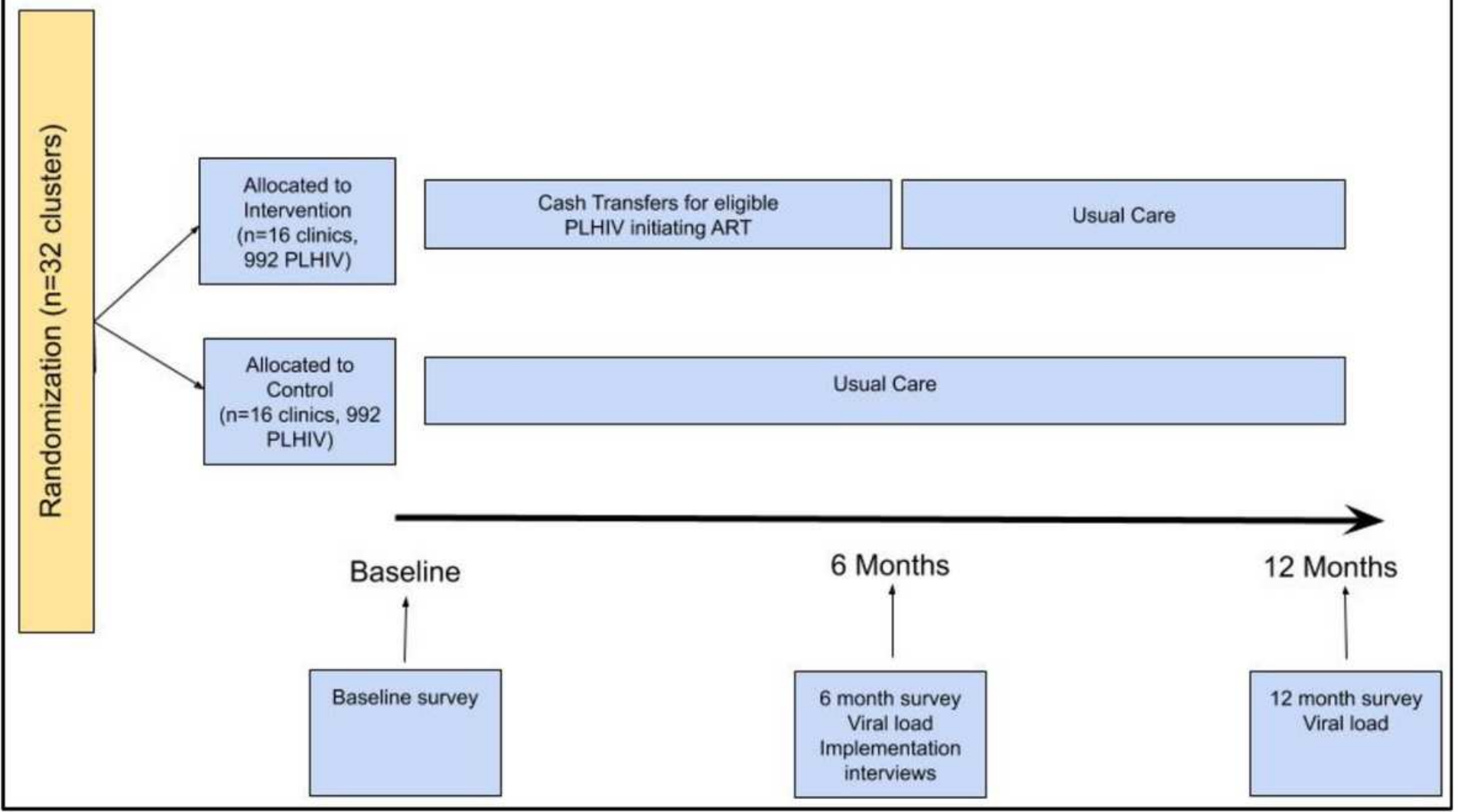

Figure 1

\section{Supplementary Files}

This is a list of supplementary files associated with this preprint. Click to download.

- CONSORTExtensionforClusterTrialsAfya2Phase2.pdf 